\title{
Nuances of transradial approach for mechanical thombectomy in acute basilar artery occlusion
}

\author{
Leonardo Rangel-Castilla, MD, and Giuseppe Lanzino, MD
}

Departments of Neurologic Surgery and Radiology, Mayo Clinic, Rochester, Minnesota

In elderly patients with acute ischemic stroke, tortuosity of the proximal vertebral artery makes access from the transfemoral route challenging and time consuming. In such cases, a transradial approach (TA) offers a more direct vertebral artery (VA) access that overcomes proximal VA tortuosity. In this video the authors illustrate nuances of the TA for acute basilar artery occlusion in two patients with challenging proximal VA anatomy. Techniques, devices, and pitfalls are discussed. In both patients, mechanical clot retrieval was successful and resulted in significant recovery of function. The authors believe that the TA should be the initial approach for basilar artery (BA) occlusion management in elderly patients and should be considered for selected patients with other conditions requiring endovascular treatment.

The video can be found here: https://youtu.bel_Ym9tMKUy_4.

KEYWORDS basilar artery; stroke; mechanical thrombectomy; radial artery; transradial approach; video 